\title{
Stabilization of one-dimensional solitons against the critical collapse by quintic nonlinear lattices
}

\author{
Jianhua Zeng ${ }^{1,2}$ and Boris A. Malomed ${ }^{1}$ \\ ${ }^{1}$ Department of Physical Electronics, School of Electrical Engineering, \\ Faculty of Engineering, Tel Aviv University, Tel Aviv 69978, Israel \\ ${ }^{2}$ State Key Laboratory of Low Dimensional Quantum Physics, \\ Department of Physics, Tsinghua University, Beijing 100084, China
}

\begin{abstract}
It has been recently discovered that stabilization of two-dimensional (2D) solitons against the critical collapse in media with the cubic nonlinearity by means of nonlinear lattices (NLs) is a challenging problem. We address the 1D version of the problem, i.e., the nonlinear-Schrödinger equation (NLSE) with the quintic or cubic-quintic (CQ) terms, the coefficient in front of which is periodically modulated in space. The models may be realized in optics and Bose-Einstein condensates (BECs). Stability diagrams for the solitons are produced by means of numerical methods and analytical approximations. It is found that the sinusoidal NL stabilzes solitons supported by the quintic-only nonlinearity in a narrow stripe in the respective parameter plane, on the contrary to the case of the cubic nonlinearity in $2 \mathrm{D}$, where the stabilization of solitons by smooth spatial modulations is not possible at all. The stability region is much broader in the $1 \mathrm{D}$ CQ model, where higher-order solitons may be stable too.
\end{abstract}

PACS numbers: 42.65.Tg, 05.45.Yv, 03.75.Kk, 03.75.Lm

\section{INTRODUCTION}

The use of periodic potentials, induced by photonic crystals or optical lattices for controlling light beams in optical waveguides [1] or matter waves in Bose-Einstein condensates (BECs) 2] was the subject of many works, see reviews [3]-7]. It has been demonstrated that lattice potentials balancing the self-focusing or defocusing cubic nonlinearity uphold bright solitons - ordinary ones or gap modes, respectively. The general topic of solitons in period potentials has been reviewed in detail in Refs. $[3]$ - $[\underline{6}]$. In the two-dimensional (2D) geometry, where the cubic $\left(\chi^{(3)}\right)$ self-focusing leads to the critical collapse [8], fundamental solitons in free space (Townes solitons [9]) are unstable, representing a separatrix between collapsing and decaying modes. Vortex solitons in the 2D free space exist too [10], being subject to a still stronger instability against azimuthal perturbations [4, 11]). However, lattice potentials can stabilize 2D fundamental solitons and solitary vortices alike [12]. Moreover, the same potentials may also stabilize 2D solitons against the supercritical collapse, driven by the self-focusing quintic $\left(\chi^{(5)}\right)$ term [13].

In $1 \mathrm{D}$, the critical collapse is induced by the quintic nonlinearity, which gives rise to the known $1 \mathrm{D}$ counterparts of the Townes solitons, see Eq. (4) below. The analogy to the 2D setting suggests a possibility of the stabilization of the 1D variety of Townes solitons by means of the single-well or lattice potentials, which was demonstrated in Refs. [14] and [15], respectively, with the help of numerical simulations and a variational approximation (VA). As concerns physical realizations, the quintic nonlinearity (acting, generally, in a combination with the cubic term) was theoretically predicted [16] and experimentally observed in diverse optical media, including liquids [17, 18], glasses [19] and ferroelectric films 20]. Generally, this nonlinearity comes along with the cubic losses. Nevertheless, the losses may be usually neglected over relatively short propagation distances for which experiments are run. In particular, the cubic-quintic $\left(\mathrm{CQ}\right.$, alias $\left.\chi^{(3)}: \chi^{(5)}\right)$ optical nonlinearity may be efficiently adjusted in colloids formed of metallic nanoparticles, by selecting the size of the particles and the colloidal filling factor [18]. Using these parameters, one may emulate a medium with the quintic-only nonlinearity, i.e., $\chi^{(3)}=0$, which is the case especially interesting for the theoretical consideration, see below. Another realization of an effectively quintic medium may be possible in a composite material built as an alternation of narrow self-focusing and defocusing layers [21], in which the cubic $\chi^{(3)}$ coefficient is canceled on the average, while its $\chi^{(5)}$ coefficient remains uncompensated.

In terms of the BEC, the quintic term in the Gross-Pitaevskii equation (GPE) may represent three-body interactions in a sufficiently dense condensate, provided that the lossy part of these interactions may be neglected (the latter depends on particular properties of the condensate, the use of the Feshbach resonance for controlling the scattering length of inter-atomic collisions, etc.) [22]. On the other hand, a universal quintic term appears in the effective GPE, in the absence of any three-body interactions, as a result of the reduction of the dimension from 3 to 1 under the action of the strong confinement in the transverse plane [23, 24]. In the latter case, no dissipative nonlinearity appears, and the sign of the effective quintic term is self-attractive, irrespective of the sign of the scattering length which determines the self-attractive or repulsive sign of the cubic nonlinearity. The relative strength of the respective quintic term in comparison to the basic cubic one can be estimated as $\sim 5|a| n_{1 \mathrm{D}}$ (see, e.g., Ref. [24]), where $a$ is the 
scattering length ( $a<0$ corresponds to the attraction between atoms), and $n_{1 \mathrm{D}}$ the $1 \mathrm{D}$ density of the condensate. For the typical situation with matter-wave solitons containing $\sim 5000$ atoms in the area of size $\sim 2 \mu \mathrm{m}$, with $a \sim-0.1$ $\mathrm{nm}[25]$, the estimate yields the value $\sim 1$, hence the quintic terms may be as important as the cubic one.

More recently, the studies of the soliton dynamics in periodic potentials were extended for nonlinear lattices (NLs), which are represented by pseudopotentials [26] induced by spatially periodic modulations of the local strength of the nonlinearity, see original works [27]- [33] and recent review [7]. In optics and BEC, NLs may be engineered by means of various techniques, such as filling voids of photonic crystals with properly selected materials, or using the Feshbach resonance controlled by spatially patterned fields [7] (in particular, magnetic lattices governing the local strength of the Feshbach resonance have been built using sets of ferromagnetic films [29]). In quasi-1D BECs, an NL can also be induced by periodically modulating the transverse-confinement strength in the axial direction [30]. Similarly, a periodic modulation of the local nonlinearity strength in a planar optical waveguide may be created by varying the thickness of the waveguiding layer. In particular, the periodically modulated quintic-only and CQ nonlinearities, considered below, may be realized in the above-mentioned colloid layers with the periodic variation of the transverse thickness, or by applying a properly patterned magnetic field to the colloidal suspension formed by ferromagnetic particles.

The previous analysis has demonstrated that NLs readily support stable 1D solitons, but it is quite difficult, although possible, to stabilize 2D solitons against the critical collapse by means of nonlinear (pseudo)potentials. Namely, 2D sinusoidal lattices completely fail to provide for the stabilization, while shapes with sharp edges, such as circles or stripes, are able to do it [31]-[33]. Although a periodic lattice of nonlinear circles with sharp edges can support stable $2 \mathrm{D}$ solitons, they are virtually the same as those supported by a lone circle, i.e., the periodicity plays no essential role in the stabilization [32].

The results outlined above suggest to investigate the 1D counterpart of the problem, i.e., stabilization of 1D solitons by means of NLs against the respective critical collapse, accounted for by the $\chi^{(5)}$ (quintic-only) or CQ nonlinearity. Below, we perform the analysis of the model with the quintic-only and CQ self-focusing terms, whose strength is subject to the periodic spatial modulation, by means of numerical methods and analytical approximations. We find that a smooth sinusoidal $\chi^{(5)}$ NL can stabilize 1D solitons, on the contrary to the negative result in $2 \mathrm{D}$, but only in a narrow parametric region. This finding is explained with the help of an analytical approach. The sinusoidal CQ NL gives rise to a much broader stability area than its quintic counterpart, which is demonstrated by means of the VA, in addition to numerical results. A chain of nonlinear domains with sharp edges may support stable solitons in 1D as well, but it can be readily checked that, like their $2 \mathrm{D}$ counterparts, such solitons are actually supported by lone nonlinear islands, the effect of the periodicity being negligible.

\section{THE MODEL AND ANALYTICAL APPROXIMATIONS}

In terms of optical realizations, the scaled form of the underlying nonlinear Schrödinger equation (NLSE) for the field amplitude, $u(x, z)$, is

$$
i u_{z}=-\frac{1}{2} u_{x x}-\left[\varepsilon_{0}+\varepsilon_{1} \cos (2 x)\right]|u|^{2} u-\left[g_{0}+g_{1} \cos (2 x)\right]|u|^{4} u,
$$

where $\varepsilon_{0}$ and $g_{0}$ are strengths of the uniform CQ terms, $\varepsilon_{1}$ and $g_{1}$ represent the NL, and the scale of coordinate $x$ is fixed by setting the NL period to be $\pi$. Below, the remaining scaling invariance is used to fix $g_{1} \equiv 1$. The corresponding GPE for the mean-field wave function of BEC is obtained from Eq. (1) by replacing propagation distance $z$ with time $t$. A model featuring the periodic modulation of the cubic nonlinearity along the evolution variable $(z)$, rather than along $x$, was studied too, supporting stable motion of solitons in the discrete version of the 1D model [34].

Stationary solutions to Eq. (1) with propagation constant $k$ are sought for as $u(x, z)=U(x) \exp (i k z)$, where real function $U(x)$ satisfies equation

$$
k U=\frac{1}{2} U_{x x}+\left[\varepsilon_{0}+\varepsilon_{1} \cos (2 x)\right] U^{3}+\left[g_{0}+g_{1} \cos (2 x)\right] U^{5},
$$

which can be derived from the Lagrangian,

$$
\begin{aligned}
L= & \frac{1}{2} \int_{-\infty}^{+\infty}\left\{k U^{2}+\frac{1}{2} U_{x}^{2}-\frac{1}{2}\left[\varepsilon_{0}+\varepsilon_{1} \cos (2 x)\right] U^{4}\right. \\
& \left.-\frac{1}{3}\left[g_{0}+g_{1} \cos (2 x)\right] U^{6}\right\} d x .
\end{aligned}
$$


In the case of $\varepsilon_{0,1}=g_{1}=0$, the above-mentioned unstable Townes-type solution of the quintic NLSE with $g_{0}>0$ is well known:

$$
U^{2}(x)=\sqrt{3 k / g_{0}} \operatorname{sech}(\sqrt{8 k} x)
$$

whose norm (the total power or number of atoms, in terms optics or BEC, respectively) does not depend on $k$,

$$
N=\int_{-\infty}^{+\infty} U^{2}(x) d x=\frac{\pi}{2} \sqrt{\frac{3}{2 g_{0}}}
$$

which is the characteristic feature of Townes solitons [8]. The addition of an arbitrarily weak cubic self-focusing term, with constant coefficient $\varepsilon_{0}>0$, lifts the degeneracy and turns solution (4) into exact stable solutions of the CQ equation [35, 36]. The addition of the self-defocusing cubic term with $\varepsilon_{0}<0$ lifts the degeneracy too, but makes the entire soliton family unstable [24, 36].

To apply the VA [37], we adopt the Gaussian ansatz for the soliton with width $W$ and norm $N$ :

$$
U^{2}(x)=\frac{N}{\sqrt{\pi} W} \exp \left(-\frac{x^{2}}{W^{2}}\right) .
$$

The substitution of the ansatz into Lagrangian (3) yields the effective Lagrangian,

$$
L_{\mathrm{eff}}=\frac{N}{2}\left[k+\frac{1}{4 W^{2}}-\frac{N}{2 \sqrt{2 \pi} W}\left(\varepsilon_{0}+\varepsilon_{1} e^{-\frac{W^{2}}{2}}\right)-\frac{N^{2}}{3 \sqrt{3} \pi W^{2}}\left(g_{0}+g_{1} e^{-\frac{W^{2}}{3}}\right)\right],
$$

which gives rise to the corresponding variational equations, $\partial L_{\mathrm{eff}} / \partial W=\partial L_{\mathrm{eff}} / \partial N=0$ :

$$
\begin{gathered}
\frac{-1}{4 W^{2}}+\frac{N\left(\varepsilon_{0}+\varepsilon_{1} e^{-\frac{W^{2}}{2}}\right)}{\sqrt{2 \pi} W}+\frac{N^{2}\left(g_{0}+g_{1} e^{-\frac{W^{2}}{3}}\right)}{\sqrt{3} \pi W^{2}}=k, \\
\frac{-1}{2 W^{2}}+\frac{N}{2 \sqrt{2 \pi}}\left[\frac{\varepsilon_{0}}{W}+\varepsilon_{1}\left(\frac{1}{W^{2}}+W\right) e^{-\frac{W^{2}}{2}}\right] \\
+\frac{2 N^{2}}{3 \sqrt{3} \pi}\left[\frac{g_{0}}{W^{2}}+g_{1}\left(\frac{1}{W^{2}}+\frac{1}{3}\right) e^{-\frac{W^{2}}{3}}\right]=0 .
\end{gathered}
$$

As shown below, the VA produces reasonable results in the model with the CQ nonlinearity. The VA may be extended to investigate the stability of perturbed (nonstationary) solitons by including the chirp into the ansatz, but this is not really necessary, as the stability of the solitons which are reasonably well approximated by the ansatz is correctly predicted by the VK criterion, as shown below.

The VA fails to produce accurate results for the quintic-only model $\left(\varepsilon_{0,1}=0\right)$, unlike the CQ one (see below). Nevertheless, in the quintic model another analytical approximation can be applied to broad solitons with $k \lesssim 1$ and $g_{0} \gg g_{1}$. In this case, an approximate correction to soliton (4), generated by term $\sim g_{1}$ in Eq. (2), is found to be

$$
U_{1} \approx\left(g_{1} / 2\right)\left(3 k / g_{0}\right)^{5 / 4}[\operatorname{sech}(\sqrt{8 k} x)]^{5 / 2} \cos (2 x)
$$

and the corresponding correction to norm (5), which lifts its degeneracy, is

$$
N_{1} \approx(3 \sqrt{3} \pi / 4) g_{1} g_{0}^{-3 / 2} \exp (-\pi /(2 \sqrt{2 k}))
$$

Thus, correction (8) replaces the single value (5) by the exponentially narrow stripe of values (9), within which the norm satisfies the Vakhitov-Kolokolov (VK) necessary stability criterion, $d N / d k>0$ [8, 38], suggesting that there may exist a narrow stability stripe for the $1 \mathrm{D}$ solitons in the quintic-NL model. This expectation is confirmed by numerical results presented below.

For the sake of the comparison of the models with the critical (quintic and cubic, respectively) nonlinearities in 1D and $2 \mathrm{D}$, it is relevant to mention that an approximation similar to that based on Eq. (8) can be developed too for broad $2 \mathrm{D}$ solitons in the $\chi^{(3)}$ model with the full 2D or quasi-1D sinusoidal modulation of the nonlinearity, making use of formula $\int_{0}^{2 \pi} \cos (2 r \cos \theta) d \theta=2 \pi J_{0}(2 r)$ for the angular integration in the calculation of the ensuing correction for the norm, and of the asymptotic form for the $2 \mathrm{D}$ Townes soliton, $U_{0}(r) \sim r^{-1 / 2} \exp (-\sqrt{2 k} r)$. However, the eventual result for the $2 \mathrm{D}$ setting turns out to be opposite: $d N^{(2 \mathrm{D})} / d k<0$, which explains the failure to of the sinusoidal NL to stabilize the $2 \mathrm{D}$ solitons. 

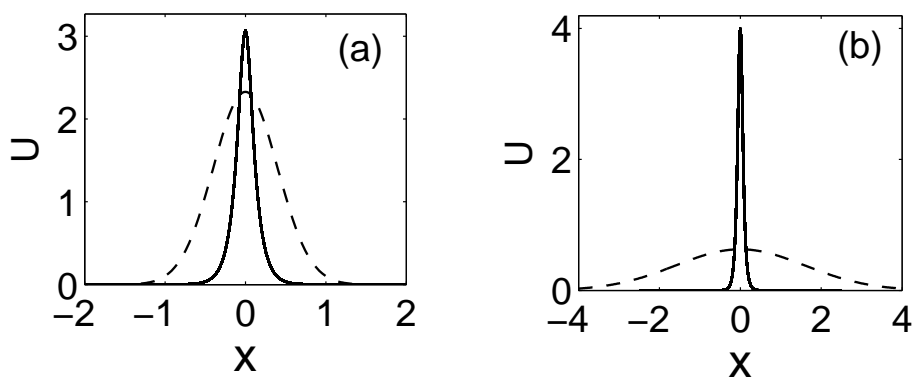

FIG. 1: Shapes of stable (a) and unstable (b) solitons in the quintic model with $\varepsilon=0, g_{0}=0.5$ are shown for $k=69.8, N=1.65$, and $k=71.5, N=1.78$, respectively. The dashed lines represent the shapes predicted by the variational approximation.


FIG. 2: (Color online) The evolution of the stable and unstable solitons from Fig. 1 is displayed in the left and central panels, respectively. In addition, the right panel shows an example of the unstable evolution towards the collapse in the quintic model (unlike the decay in the central panel), for the soliton with $k=88.2$ and $N=1.85$.

\section{LOCALIZED MODES IN THE QUINTIC MODEL}

Stationary solutions to Eq. (11), i.e., solutions of Eq. (2), were found by means of the imaginary-time-propagation method [39], and their stability was tested by subsequent simulations of the real propagation.

Examples of the shape and evolution of stable and unstable solitons supported by the quintic-only $\left(\varepsilon_{0,1}=0\right) \mathrm{NL}$ are displayed in Figs. 11and 2, In Fig. 1, the comparison with the numerical findings demonstrates that the Gaussian ansatz fails to correctly predict the actual shape of the solitons in the case of the quintic nonlinearity, therefore only numerical results are reported in this section. For very narrow solitons, whose width is much smaller than the NL period [such as the soliton shown in Fig. 1(b)], Eq. (2) is virtually tantamount to the equation with the constant nonlinearity coefficient, $\tilde{g}_{0} \equiv g_{0}+g_{1}=g_{0}+1$ (recall we fix $g_{1} \equiv 1$ ), and, accordingly, the narrow solitons may be considered as Townes modes (4), with $g_{0}$ replaced by $\tilde{g}_{0}$. Therefore, the narrow solitons are unstable, see the central and right panels in Fig. 2. The instability manifests itself as a quick decay of the soliton (see the central panel in Fig. 2), or as the evolution towards the collapse, see the right panel in Fig. 2, Note the great difference in the scale of $z$ in the panels of Fig. 2 demonstrating the stable and unstable evolution (i.e., the stable behavior persists indefinitely long, while the instability sets in quickly). It is relevant to compare these scales with the diffraction length corresponding to the solitons displayed in Fig. 1, which is $z_{\text {diffr }} \sim 0.1$. On the other hand, it is relevant to stress that the GPE in the form of Eq. (11) may predict only the onset of the collapse, while its further development requires a more accurate (microscopic) description, in terms of the Hartree-Fock-Bogoliubov equations [40].

The numerically found dependence $k(N)$ for the solitons in the quintic model is displayed in Fig. 3 for two different values of $g_{0}$. While these curves obey the VK criterion, direct simulations of the perturbed evolution of the solitons demonstrate that the solitons are stable only in narrow intervals of the values of $N$ (recall that the VK criterion is only necessary, but not sufficient, for the stability).

The numerically found stability region of solitons in the quintic model is located between the solid borders in Fig. 4. for both positive and negative (self-focusing and defocusing) values of the constant quintic coefficient, $g_{0} \gtrless 0$. The 

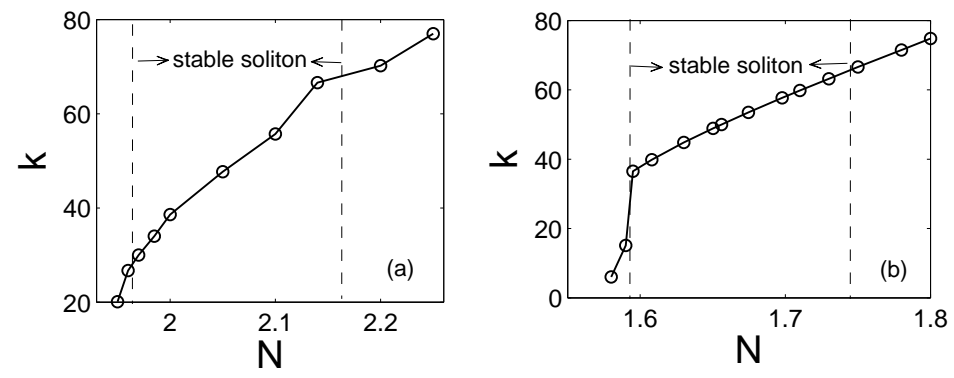

FIG. 3: The propagation constant vs. the norm for the soliton families in the quintic model $\left(\varepsilon_{0,1}=0\right)$ for $g_{0}=0(\mathrm{a})$ and $g_{0}=0.5(\mathrm{~b})$. The designated stability intervals were identified via direct simulations.
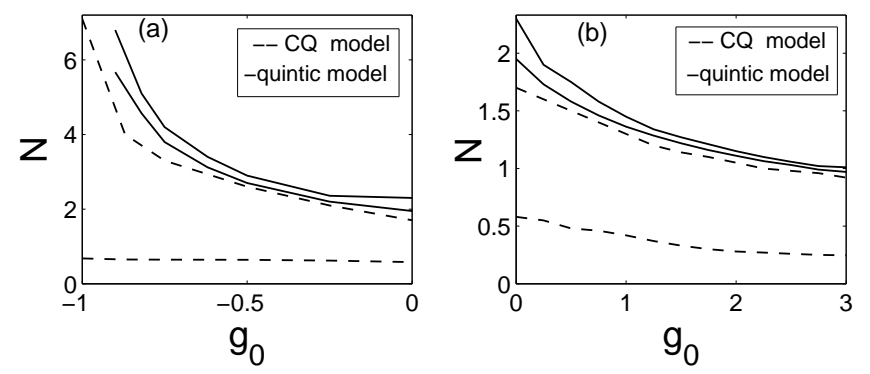

FIG. 4: Stability regions (located between the borders) for the solitons in the parameter plane of the quintic and CQ models (solid and dashed borders, respectively), shown separately for the self-defocusing (a) and self-focusing (b) sign of the constant part of the quintic coefficient, $g_{0}$.

overall shape of the narrow region is quite close to

$$
N=(\pi / 2) \sqrt{3 /\left[2\left(1+g_{0}\right)\right]},
$$

which is explained by the fact that the solitons are always found near values of $N$ corresponding to the critical one, given by Eq. (5), with $g_{0}$ substituted by the above-mentioned local value, $\tilde{g}_{0} \equiv g_{0}+1$. This argument also explains nonexistence of solitons at $g_{0} \leq-1$. The shrinkage of the stability stripe at large positive $g_{0}$ is a manifestation of the relative weakness of the NL in this limit, and is consistent with the exponential weakness of the NL-induced stabilization predicted by the above analysis, see Eq. (9). Above the narrow stripe, the unstable solitons suffer the collapse, while below the stripe they decay, which may be realized as the delocalization transition, similar to that studied in systems with linear lattice potentials [12, 41].

\section{LOCALIZED MODES IN THE CQ (CUBIC-QUINTIC) MODEL}

Unlike the model with the quintic-only nonlinearity, the one with the CQ terms gives rise to broad solitons, as shown in Fig. 5 [see also Fig. 7(a) below]. Due to this fact, the predictions produced by the VA for the CQ model are in reasonable agreement with the numerical findings, as can be seen in Figs. 5 and 6. On the other hand, the agreement deteriorates at large values of $N$, when the quintic term dominates, driving the system to the regime considered in the previous section.

Dependences $k(N)$ in Fig. [6] satisfy the VK criterion, and simulations demonstrate that the solitons in the CQ model, in contrast to the quintic-only one, are always stable when they obey the criterion. Accordingly, the stability

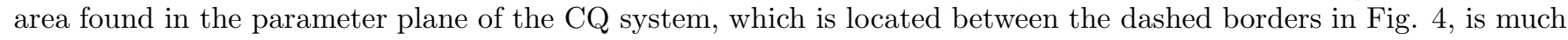
larger than its counterpart in the quintic model. The upper boundary of the area, which is determined by the collapse driven by the local quintic nonlinearity, is approximated by Eq. (10).

Another essential difference from the quintic-only model is that the stability area in the CQ system extends to $g_{0}<-1$, where the quintic nonlinearity is completely defocusing, but the solitons can be supported by the cubic 

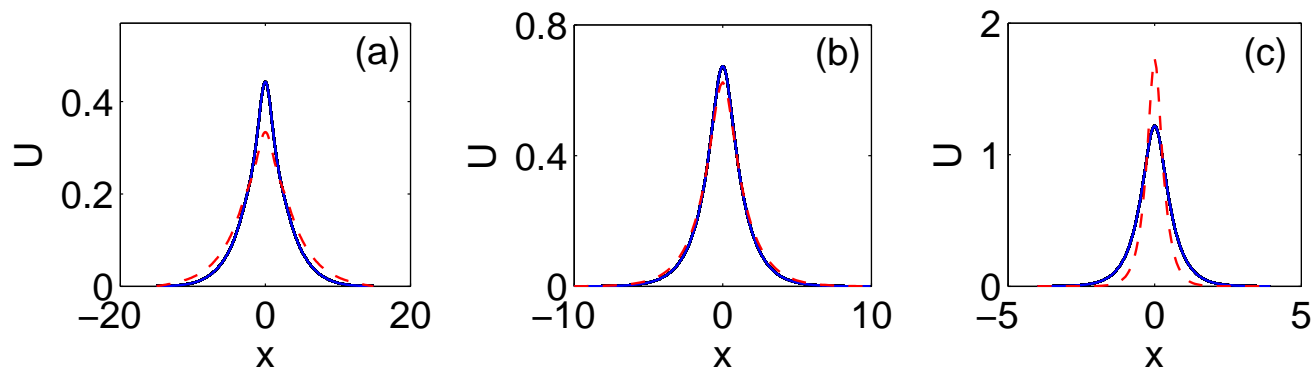

FIG. 5: (Color online) Typical examples of broad stable solitons in the CQ model with $g_{0}=0.25, \varepsilon_{0}=\varepsilon_{1}=g_{1}=1$ : (a) $k=0.26, N=0.65$; (b) $k=0.57, N=0.8$; (c) $k=3.2, N=1.2$. The dashed lines represent shapes predicted by the variational approximation for the same values of the parameters.
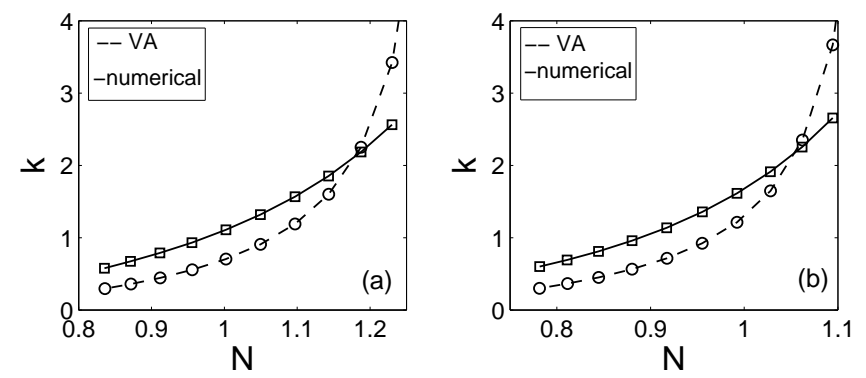

FIG. 6: Comparison of the variationally predicted and numerically found dependences $k(N)$ in the CQ model, with $\varepsilon_{0}=\varepsilon_{1}=$ $g_{1}=1$, for $g_{0}=0(\mathrm{a})$ and $g_{0}=0.5(\mathrm{~b})$.

term. In this case, stable solitons develop side peaks, as shown in Fig. 7, and form odd-numbered stable in-phase bound states, see examples of three-, five- and seven-soliton complexes in Fig. 8. The transition from the higher-order soliton, featuring the side peaks, to the bound complexes occurs when the height of the additional peaks becomes equal to that of the central maximum. Bound states of three in-phase solitons were previously found in the 1D cubic-NL model, but they were completely unstable [27].

The present system also supports stable dipoles, i.e., bound states of two solitons with opposite signs, as well as stable dipolar complexes built of two multi-peak soliton clusters, as shown in Fig. 9. The simplest two-peak dipoles are similar to their counterparts found in the cubic-NL model [27].


FIG. 7: Examples of a stable broad soliton (a) and stable solitons with symmetric side peaks (b, c) in the CQ model with $\varepsilon_{0}=\varepsilon_{1}=g_{1}=1$ and $g_{0}=-1.05$ : (a) $k=2.88, N=19.5$; (b) $k=5.22, N=21.3$; (c) $k=9.23, N=22$. 

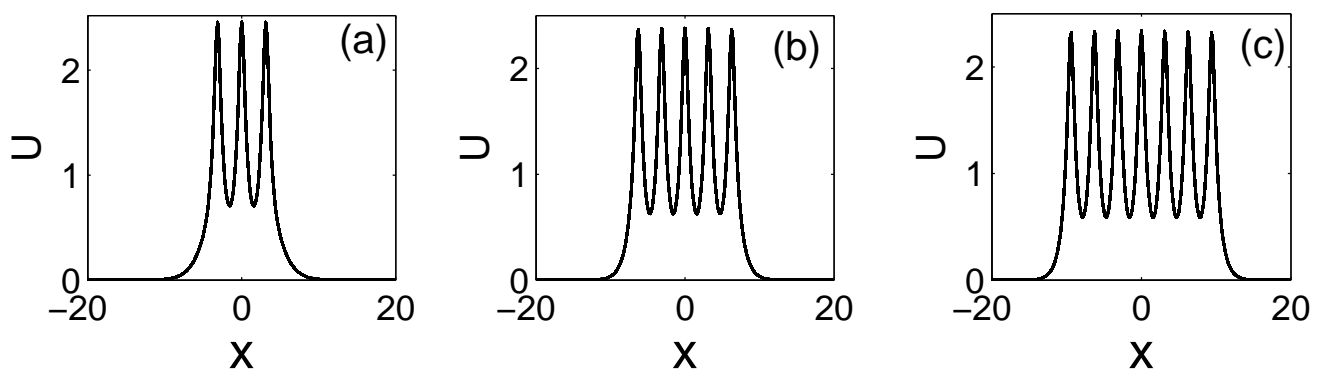

FIG. 8: Examples of stable in-phase bound states of three (a), five (b), and seven (c) solitons in the CQ model with $\varepsilon_{0}=\varepsilon_{1}=$ $g_{1}=1$, and $g_{0}=-1.25$ : (a) $k=0.73, N=20$; (b) $k=1.12, N=30$; (c) $k=0.73, N=40$.
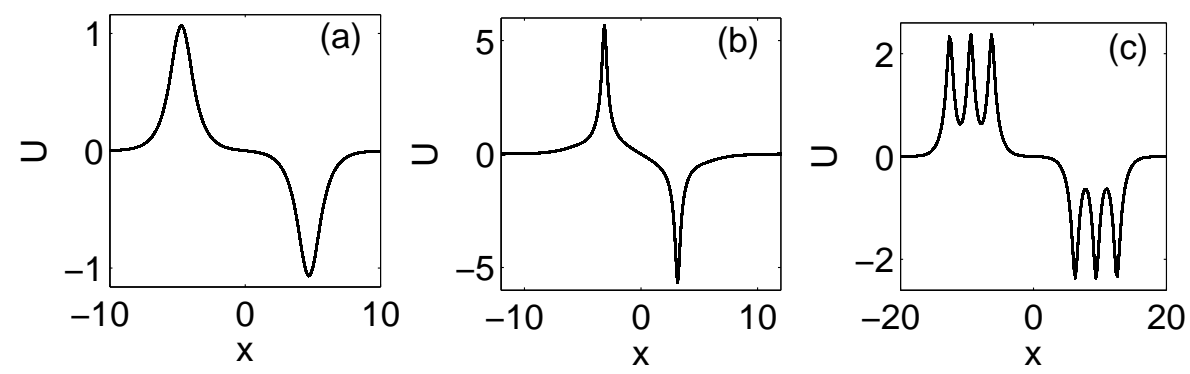

FIG. 9: Examples of stable dipole solitons (a, b), and of a stable cluster dipole (c), in the CQ model with $\varepsilon_{0}=\varepsilon_{1}=1$. Parameters are: (a) $g_{0}=0.5, k=1.1, N=3.4$; (b) $g_{0}=-1.05, k=2, N=38$; (c) $g_{0}=-1.25, k=1.1, N=36$. Note that the quintic term is self-defocusing in cases (b) and (c), but self-focusing, on the average, in (a).

\section{CONCLUSIONS}

This work aims to introduce the smooth sinusoidal NL (nonlinear lattice) acting on the critical quintic $\left(\chi^{(5)}\right)$ nonlinearity in the 1D setting, as well as the system with the CQ (cubic-quintic) NL. The models approximate settings which are relevant to optics and BEC. Stability regions for solitons in these systems have been identified, being narrow in the quintic-only model, and much broader in the CQ one. In the latter case, the results may be predicted analytically by means of the VA (variational approximation), in the combination with the VK stability criterion. The narrow stability stripe in the quintic model, and its absence in the recently studied 2D models with sinusoidal NLs acting on the cubic nonlinearity (which is critical in the 2D geometry) were also qualitatively explained by means of an analytical approximation. A notable feature of the CQ NL is that it generates stable broad solitons, which may develop side peaks and form stable bound states, both in-phase and out-of-phase ones.

It may be interesting to study other dynamical features in models of this type, such as mobility of the solitons. A relevant extension should also be consideration of two-component systems similar to the scalar models introduced in this work, cf. the analysis of two-component solitons in the cubic-NL system, which was reported in Ref. [42].

Acknowledgments. The work of J.Z. was supported by a postdoctoral fellowship provided by the Tel Aviv University, and by grant No. 149/2006 from the German-Israel Foundation.

[1] J. D. Joannopoulos, S. G. Johnson, J. N. Winn, and R. D. Meade, Photonic Crystals: Molding the Flow of Light (Princeton University Press: Princeton, 2008).

[2] C. J. Pethick and H. Smith, Bose-Einstein condensate in dilute gas (Cambridge University Press: Cambridge, 2008).

[3] V. A. Brazhnyi, and V. V. Konotop, Mod. Phys. Lett. B 18, 627 (2004); O. Morsch and M. Oberthaler, Rev. Mod. Phys. 78, 179 (2006).

[4] B. A. Malomed, D. Mihalache, F. Wise, and L. Torner, J. Optics B: Quant. Semicl. Opt. 7, R53 (2005).

[5] F. Lederer, G. I. Stegeman, D. N. Christodoulides, G. Assanto, M. Segev, and Y. Silberberg, Phys. Rep. 463, 1 (2008). 
[6] Y. V. Kartashov, V. A. Vysloukh, and L. Torner, Progress in Optics 52, 63 (ed. by E. Wolf: North Holland, Amsterdam, 2009).

[7] Y. V. Kartashov, B. A. Malomed, and L. Torner, Rev. Mod. Phys. 83, 247 (2011).

[8] L. Bergé, Phys. Rep. 303, 259 (1998).

[9] R. Y. Chiao, E. Garmire, and C. H. Townes, Phys. Rev. Lett. 13, 479 (1964).

[10] V. I. Kruglov, Y. A. Logvin, and V. M. Volkov, J. Mod. Opt. 39, 2277 (1992).

[11] A. I. Yakimenko, Yu. A. Zaliznyak, and Yu. S. Kivshar, Phys. Rev. E 71, 065603(R) (2005).

[12] B. B. Baizakov, B. A. Malomed, and M. Salerno, Europhys. Lett. 63, 642 (2003); Phys. Rev. A 70, 053613 (2004); J. Yang and Z. H. Musslimani, Opt. Lett. 28, 2094 (2003).

[13] R. Driben and B. A. Malomed, Eur. Phys. J. D 50, 317 (2008).

[14] Yu. B. Gaididei, J. Schjodt-Eriksen, and P. L. Christiansen, Phys. Rev. E 60, 4877 (1999).

[15] F. Kh. Abdullaev and M. Salerno, Phys. Rev. A 72, 033617 (2005); G. L. Alfimov, V. V. Konotop, and P. Pacciani, Phys. Rev. A 75, 023624 (2007).

[16] G. S. Agarwal and S. Dutta Gupta, Phys. Rev. A 38, 5678 (1988); K. Dolgaleva, R. W. Boyd, J. E. Sipe, Phys. Rev. A 76, 063806 (2007).

[17] C. Zhan, D. Zhang, D. Zhu, D. Wang, Y. Li, D. Li, Z. Lu, L. Zhao, and Y. Nie, J. Opt. Soc. Am. B 19, 369 (2002); R. A. Ganeev, M. Baba, M. Morita, A. I. Ryasnyansky, M. Suzuki, M. Turu, H. Kuroda, J. Opt. A: Pure Appl. Opt. 6, 282 (2004).

[18] E. L. Falcão-Filho, C. B. de Araújo, and J. J. Rodrigues Jr., J. Opt. Soc. Am. B 24, 2948 (2007).

[19] C. Zhan, D. Zhang, D. Zhu, D. Wang, Y. Li, D. Li, Z. Lu, L. Zhao, and Y. Nie, J. Opt. Soc. Am. B 19, 369 (2002); G. Boudebs, S. Cherukulappurath, H. Leblond, J. Troles, F. Smektala, and F. Sanchez, Opt. Commun. 219 , 427 (2003); F. Smektala, C. Quemard, V. Couderc, and A. Barthélémy, J. Non-Cryst. Solids 274, 232 2000; K. Ogusu, J. Yamasaki, S. Maeda, M. Kitao, and M. Minakata, Opt. Lett. 29, 265 (2004); F. Sanchez, G. Boudebs, S. Cherukulappurath, H. Leblond, J. Troles, and F. Smektala, J. Nonlinear Opt. Phys. Mater. 13, 7 (2004).

[20] B. Gu, Y. Wang, W. Ji, and J. Wang, Appl. Phys. Lett. 95, 041114 (2009).

[21] I. Towers and B. A. Malomed, J. Opt. Soc. Am. 19, 537 (2002).

[22] P. F. Bedaque, E. Braaten, and H. W. Hammer, Phys. Rev. Lett. 85, 908 (2000); A. Gammal, T. Frederico, L. Tomio, and F. K. Abdullaev, Phys. Lett. A 267, 305 (2000); F. Kh. Abdullaev, A. Gammal, L. Tomio, and T. Frederico, Phys. Rev. A 63, 043604 (2001); E. Braaten, H. W. Hammer, and T. Mehan, Phys. Rev. Lett. 88, 040401 (2002); J. W. Michael, ibid. 89, 140402 (2002); W. Zhang, E. M. Wright, H. Pu, and P. Meystre, Phys. Rev. A 68, 023605 (2003); E. Fersino, G. Mussardo, and A. Trombettoni, ibid. 77, 053608 (2008).

[23] A. E. Muryshev, G. V. Shlyapnikov, W. Ertmer, K. Sengstock, and M. Lewenstein, Phys. Rev. Lett. 89, 110401 (2002); L. Salasnich, A. Parola, and L. Reatto, Phys. Rev. A 65, 043614 (2002); ibid. 66, 043603 (2002); L. D. Carr and J. Brand, Phys. Rev. Lett. 92, 040401 (2004); Phys. Rev. A 70, 033607 (2004).

[24] L. Khaykovich and B. A. Malomed, Phys. Rev. A 74, 023607 (2006).

[25] K. E. Strecker, G. B. Partridge, A. G. Truscott, and R. G. Hulet, New J. Phys. 5, 73.1 (2003).

[26] T. Mayteevarunyoo, B. A. Malomed, and G. Dong, Phys. Rev. A 78, 053601 (2008).

[27] H. Sakaguchi and B. A. Malomed, Phys. Rev. E 72,046610 (2005).

[28] G. Theocharis, P. Schmelcher, P. G. Kevrekidis, and D. J. Frantzeskakis, Phys. Rev. E 72, 033614; F. Kh. Abdullaev and J. Garnier, ibid. 72,061605(R) (2005); Y. Sivan, G. Fibich, and M. I. Weinstein, Phys. Rev. Lett. 97, 193902 (2006); Z. Rapti, P. G. Kevrekidis, V. V. Konotop, and C. K. R. T. Jones, J. Phys. A 40, 14151 (2007).

[29] S. Ghanbari, T. D. Kieu, A. Sidorov, and P. Hannaford, J. Phys. B: At. Mol. Opt. Phys. 39, 847 (2006); A. Abdelrahman, P. Hannaford, and K. Alameh, Opt. Exp. 17, 24358 (2009).

[30] L. Salasnich, A. Cetoli, B. A. Malomed, F. Toigo, and L. Reatto, Phys. Rev. A 76, 013623 (2007).

[31] H. Sakaguchi and B. A. Malomed, Phys. Rev. E 73, 026601 (2006).

[32] Y. V. Kartashov, B. A. Malomed, V. A. Vysloukh, and L. Torner, Opt. Lett. 34, 770 (2009).

[33] Y. Sivan, G. Fibich, B. Ilan, and M. I. Weinstein, Phys. Rev. E 78, 046602 (2008); N. V. Hung, P. Ziń, M. Trippenbach, and B. A. Malomed, ibid. 82, 046602 (2010); T. Mayteevarunyoo, B. A. Malomed, and A. Reoksabutr, J. Mod. Opt. 58, 1977 (2011).

[34] J. Cuevas, B. A. Malomed and P. G. Kevrekidis, Phys. Rev. E 71, 066614 (2005).

[35] K. I. Pushkarov, D. I. Pushkarov, and I. V. Tomov, Opt. Quantum Electron. 11, 471 (1979).

[36] D. E. Pelinovsky, Y. S. Kivshar and V. V. Afanasjev, Physica D 116, 121 (1998).

[37] B. A. Malomed, Progress in Optics 43, 71 (ed. by E. Wolf: North Holland, Amsterdam, 2002).

[38] M. Vakhitov and A. Kolokolov, Radiophys. Quantum. Electron. 16, 783 (1973).

[39] W. Bao and Q. Du, SIAM J. Sci. Comput. 25, 1674 (2004).

[40] S. Wuster, B. J. Dabrowska-Wuster, A. S. Bradley, M. J. Davis, P. B. Blakie, J. J. Hope, and C. M. Savage, Phys. Rev. A 75, 043611 (2007).

[41] B. B. Baizakov and M. Salerno, Phys. Rev. A 69, 013602 (2004).

[42] Y. V. Kartashov, B. A. Malomed, V. A. Vysloukh, and L. Torner, Opt. Lett. 34, 3625 (2009). 